\title{
DETERMINAÇÃO DE ISOFLAVONAS EM DERIVADOS DE SOJA ${ }^{1}$
}

\author{
Maria Inés GENOVESE ${ }^{2}$, , \\ Franco M. LAJOLO ${ }^{3}$
}

\begin{abstract}
RESUMO
O trabalho teve como objetivo a determinação de metodologias adequadas de extração e análise de isoflavonas de derivados protéicos de soja e alimentos industrializados. Compararam-se dois métodos de determinação de isoflavonas, um baseado na análise dos diversos glicosil-derivados naturalmente presentes, e o outro, na análise de agliconas, após hidrólise ácida. Avaliaram-se também as condições ideais para extração em fase sólida. Os resultados mostraram ser o metanol aquoso a $80 \%$ o melhor meio extrator em termos de rendimento e perfil obtido, e a temperatura de $40^{\circ} \mathrm{C}$ durante a rotaevaporação adequada para a concentração dos extratos. Não houve diferenças significativas nos resultados obtidos utilizando-se filtros de 0,45 ou $0,22 \mathrm{~mm}$. Os testes de recuperação resultaram em valores entre 95,4 e 103,3\%, dependendo do composto. A hidrólise ácida para análise de agliconas mostrou-se inadequada por causar diminuição significativa dos teores de isoflavonas. A poliamida mostrou-se uma fase sólida eficiente para ligação de isoflavonas, sendo que para a eluição de malonilglicosídeos é necessário aumentar o pH do metanol.
\end{abstract}

Palavras-chave: soja; derivados; isoflavonas; determinação; extração em fase sólida.

\section{SUMMARY}

DETERMINATION OF ISOFLAVONES IN SOY PRODUCT. The objective of this work was to evaluate the conditions for extraction and analysis of isoflavones in soy products by HPLC. Methods based on isoflavone quantitation as aglycones (after acid hydrolysis) were compared to those based on quantitation of naturally occurring glucosides. Best conditions for solid phase extraction of isoflavones were also determined. Results showed $80 \%$ aqueous methanol an efficient extractant and the temperature of $40^{\circ} \mathrm{C}$ adequate for extract concentration using a rotary evaporator. Sample filtration through 0.22 or $0.45 \mathrm{~mm}$ polytetrafluoroethylene filter units had no influence on isoflavone quantitation results. Spiking recovery values were between 95.4 and $103.3 \%$, depending on the compound. Acid hydrolysis for the determination of isoflavones as their respective aglycones was shown to be inadequate for leading to significant isoflavone losses. Polyamide was efficient for solid phase extraction of isoflavones, but for complete elution of conjugates $\mathrm{pH}$ of methanol had to be increased.

Keywords: soy products; isoflavones; determination; solid phase extraction.

\section{1 - INTRODUÇÃO}

As plantas produzem uma ampla variedade de compostos de baixo peso molecular denominados metabólitos

${ }^{1}$ Recebido para publicação em 03/10/00. Aceito para publicação em 18/04/01.

${ }^{2}$ Prof. Doutor Ciência dos Alimentos. e-mail: genovese@usp.br ${ }^{3}$ Prof. Titular Ciência dos Alimentos. Depto. de Alimentos e Nutrição Experimental - Fac. Ciências Farmacêuticas. USP. Conj. Químicas, Bloco 14. Av. Lineu Prestes, 580. CEP 05508-900. São Paulo - SP. *A quem a correspondência deve ser enviada. secundários, entre os quais encontram-se os chamados fitoquímicos, tais como flavonóides, ácidos fenólicos, saponinas, glicosinolatos, glicoalcalóides, furanocumarinas e compostos cianogênicos. Os fitoquímicos possuem diversas funções na planta, principalmente de defesa contra 0 ataque de predadores e patógenos. Quando presentes em alimentos de origem vegetal, podem ter tanto efeitos benéficos como prejudiciais ao ser humano [8]. As isoflavonas são as formas mais comuns de fitoestrógenos, sendo predominantemente encontradas em leguminosas e especialmente abundantes na soja [11]. As isoflavonas possuem estrutura química semelhante à dos estrógenos, tais como o 17 b-estradiol. Dados experimentais e clínicos têm mostrado que as isoflavonas representam uma alternativa promissora na prevenção e/ou tratamento de muitas doenças hormônio-dependentes, incluindo câncer, sintomas da menopausa, doenças cardiovasculares e osteoporose [9]. A soja participa da dieta humana através do consumo do próprio grão e de alimentos elaborados a partir destes, tais como tofu, miso e tempeh. Os derivados protéicos de soja, tais como farinhas desengorduradas, texturizados, concentrados e isolados protéicos, são também utilizados na produção de diversos alimentos industrializados, tais como produtos cárneos, produtos de panificação, molhos e sopas. No entanto, para a elaboração de recomendações nutricionais é necessário o conhecimento dos teores dessas substâncias nos alimentos, o qual depende do desenvolvimento de metodologias adequadas de extração e análise.

Com esse objetivo, avaliamos as melhores condições para extração das isoflavonas da soja e derivados, e para preparo da amostra para análise através de cromatografia líquida de alta eficiência (CLAE). Comparamos também os teores obtidos através da determinação das isoflavonas na forma de agliconas (após hidrólise ácida) e na forma dos glicosil-derivados originalmente presentes no produto. Com a finalidade de analisar alimentos industrializados, nos quais esperam-se teores reduzidos de isoflavonas, otimizamos as condições de extração em fase sólida, como forma de purificação e concentração desses compostos.

\section{2 - MATERIAIS E MÉTODOS}

\section{1 - Materiais}

Os derivados de soja foram obtidos industrialmente pela Ceval Alimentos S.A. (Esteio, RS). Foram analisadas amostras de farinhas desengorduradas de soja (lotes A, B, C e D), isolados protéicos de soja (lotes A, $B, C$ e D) e proteína texturizada de soja. Todos os 
reagentes e solventes utilizados foram de grau analítico ou para cromatografia líquida.

\section{2 - Métodos}

\subsection{1 - Extração de isoflavonas}

As amostras foram extraídas com agitador mecânico e barra magnética por 2 horas a $4^{\circ} \mathrm{C}$, na proporção de 1:20 (m/v). Os solventes testados foram: metanol $70 \%$, metanol $80 \%$, acetonitrila $60 \%$ e acetonitrila $80 \%$. Foi realizada, também através de agitação mecânica por 2 horas a $4^{\circ} \mathrm{C}$, extração em meio ácido utilizando acetonitrila ou acetona, como sugerido por (a) WANG \& MURPHY [13], (b) SONG et al. [10] e (c) FARMAKALIDIS \& MURPHY [3], nas seguintes condições: a) $1 \mathrm{~g}$ amostra : $10 \mathrm{~mL} \mathrm{CH}_{3} \mathrm{CN}: 2 \mathrm{~mL} \mathrm{HCl} 0,1 \mathrm{~N}$; b) $1 \mathrm{~g}$ amostra : $10 \mathrm{~mL} \mathrm{CH}_{3} \mathrm{CN}: 2 \mathrm{~mL} \mathrm{HCl} \mathrm{0,1} \mathrm{N} \mathrm{:} 7 \mathrm{~mL} \mathrm{H}_{2} \mathrm{O}$; c) $1 \mathrm{~g}$ amostra : $10 \mathrm{~mL}$ acetona : $2 \mathrm{~mL} \mathrm{HCl} 0,1 \mathrm{~N}$.

Além da extração usando-se agitador e barra magnética também foi realizada extração em Ultra-Turrax (Polytron® - Kinematica GmbH, Kriens-Luzern, Suiça), por 1 minuto em velocidade 5, em banho de gelo, utilizando-se uma proporção de 1:10 amostra:solvente $(\mathrm{m} / \mathrm{v})$. 0 resíduo foi re-extraído mais 2 vezes. Os extratos obtidos foram filtrados utilizando-se papel de filtro Whatman $\mathrm{n}^{\circ}$ 6. Para concentração dos extratos foi utilizado rotaevaporador (Rotavapor® RE 120 - Büchi, Flawil, Suiça), em temperaturas de banho de $40^{\circ} \mathrm{C}$, e as amostras foram concentradas até diferentes volumes, para avaliar o efeito do tempo de rotaevaporação. As amostras concentradas tiveram então seu volume ajustado com metanol e foram filtradas utilizando-se filtros de polietileno com membrana PTFE (Millipore Ltd., Bedford, E.U.A.), de 0,22 ou $0,45 \mathrm{~m}$ de poro. As extrações foram realizadas em triplicata.

\subsection{2 - Cromatografia líquida de alta eficiência}

A separação das isoflavonas foi realizada em coluna C18 NovaPak (Waters, Milford, E.U.A.) de acordo com o método de SONG et al [10]. O cromatógrafo líquido utilizado foi o da Hewlett Packard (Palo Alto, E.U.A.) série 1100 , equipado com detetor com arranjo de diodo (DAD). Os padrões de daidzeína e genisteína foram obtidos da Sigma Chemicals Co. (St. Louis, E.U.A.), e daidzina e genistina foram obtidos da Apin Chemicals Ltd. (Abingdon, Reino Unido). A identificação foi feita a partir dos tempos de retenção e dos espectros. As amostras foram injetadas em duplicata. Os resultados foram expressos como $\mathrm{mg}$ de isoflavona por $100 \mathrm{~g}$ de amostra (b.u.), após normalização das diferenças de peso molecular das formas glicosiladas, feita multiplicando-se a massa de cada derivado pela razão entre o peso molecular da respectiva aglicona e o peso molecular da forma glicosilada, conforme SONG et al [10].

\subsection{3 - Teste de recuperação}

Realizou-se teste de recuperação para os compostos daidzina e genistina, adicionados em amostras de farinha desengordurada e isolado protéico de soja, a seguir extraídas com agitador mecânico e barra magnética por 2 horas a $4^{\circ} \mathrm{C}$, na proporção de 1:20 (m/v) amostra:metanol $80 \%$. Os extratos foram concentrados em rotaevaporador $\left(40^{\circ} \mathrm{C}\right)$ até redução de seu volume em oito vezes. $O$ volume foi completado com metanol de forma a obter concentração final de $50 \%$. A recuperação (\%) foi calculada a partir da diferença entre a massa de daidzina ou genistina encontrada na amostra adicionada e a massa encontrada na amostra nãoadicionada, em relação à quantidade adicionada.

\subsection{4 - Hidrólise ácida}

A hidrólise das isoflavonas para obtenção das agliconas foi realizada de duas maneiras:

a ) hidrólise da amostra em $\mathrm{HCl} 1 \mathrm{~N}$ aquoso, por refluxo durante 2 horas em banho-maria fervente, e posterior extração das agliconas em acetonitrila, de acordo com WANG et al [12];

b ) extração e hidrólise concomitantes, com $\mathrm{HCl} 2 \mathrm{~N}$ em etanol $80 \%$, por refluxo durante 2 horas em banhomaria fervente, de acordo com FRANKE et al [4];

c ) hidrólise do extrato metanólico ou etanólico (80\%), com $\mathrm{HCl} 2 \mathrm{~N}$, em refluxo por 1-2 horas em banho-maria fervente, de acordo com PETERSSON \& KIESSLING [7].

O sistema de refluxo utilizado consistiu de condensador de vidro tipo Allihn com camisa de $40 \mathrm{~cm}$ de comprimento.

\subsection{5 - Extração em fase sólida}

Para a otimização da extração em fase sólida de isoflavonas foram testados dois sistemas. No primeiro, utilizaram-se colunas de $1 \mathrm{~g}$ de Poliamida (CC 6, Macherey-Nagel GmbH \& Co., Alemanha), preparadas em nosso laboratório, em seringas próprias de $6 \mathrm{ml}$ da HPLC Technology Co. Ltd. (Hertfordshire, Reino Unido). No segundo, utilizaram-se colunas prontas $(1 \mathrm{~g} / 6$ $\mathrm{mL}$ ) de C18 (Tech Elut SPE, HPLC Technology Co. Ltd.). As colunas foram pré-condicionadas pela passagem de $20 \mathrm{~mL}$ de metanol e $60 \mathrm{~mL}$ de água. O metanol dos extratos dos produtos analisados foi previamente eliminado através de rotaevaporação. Após a passagem dos extratos aquosos, as colunas foram lavadas com $20 \mathrm{~mL}$ de água e a eluição das isoflavonas foi feita com $50 \mathrm{~mL}$ de metanol, no caso de agliconas, ou $50 \mathrm{~mL}$ de metanol:amônia $(99,5: 0,5)$, no caso de glicosil-derivados. Utilizou-se manifold Visiprep 24DL da Supelco (Bellefonte, E.U.A.).

\subsection{6 - Análise estatística dos resultados}

Para a análise estatística dos resultados utilizou-se o programa Statistica versão 5.0 da StatSoft (Tulsa, E.U.A.). A comparação das médias foi realizada pelo teste t de Student $(P<0,05)$. 


\section{3 - RESULTADOS E DISCUSSÃO}

\section{1 - Condições de extração}

As isoflavonas da soja, daidzeína, genisteína e gliciteína, estão presentes no grão na forma de 7-O-bglicosídeos (daidzina, genistina e glicitina), 7-0-b-(6"'$O$-acetil)-glicosídeos (acetildaidzina, acetilgenistina e acetilglicitina), e 7-O-b-(6"'-O-malonil)-glicosídeos (malonildaizina, malonilgenistina e malonilglicitina), sendo que malonilgenistina e malonildaidzina correspondem juntas a cerca de $66 \%$ do total [5]. Os dados referentes ao melhor solvente para a extração de isoflavonas são conflitantes. WANG \& MURPHY [13] realizaram a extração de isolado protéico de soja com $\mathrm{CH}_{3} \mathrm{CN}: \mathrm{HCl}$ $0,1 \mathrm{~N}(5: 1)$, durante 2 horas à temperatura ambiente. Já SONG et al [10] relataram que a adição de água a esse

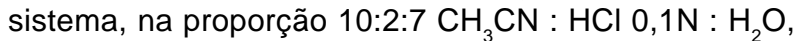
resultava em melhores recuperações. Murphy et al [6], por sua vez, indicaram que a quantidade de água a ser adicionada deveria ser otimizada para cada produto. Já FARMAKALIDIS \& MURPHY [3] utilizaram acetona: $\mathrm{HCI}$ $0,1 \mathrm{~N}$ para a extração de isoflavonas. BARNES, KIRK \& COWARD [1] encontraram a mesma eficiência de extração para metanol aquoso a $80 \%$ e acetonitrila aquosa a $80 \%$, contendo $0,1 \%$ de $\mathrm{HCl}$ (por 2 horas à temperatura ambiente). Os autores encontraram também a mesma eficiência de extração para a temperatura de $60^{\circ} \mathrm{C}$ e ambiente. No entanto, relataram que o aumento da temperatura provocava alteração na composição dos glicosil-derivados, com diminuição dos malonilglicosídeos e aumento concomitante dos b-glicosídeos. Resultados similares foram relatados por KUDOU et al [5], que compararam a extração à temperatura ambiente e a $80^{\circ} \mathrm{C}$, por etanol aquoso a 70\%. COWARD et al [2] confirmaram esses resultados e ainda relataram que a temperatura de extração de $4^{\circ} \mathrm{C}$ proporcionava o mesmo rendimento que as temperaturas mais elevadas, porém com menor degradação dos malonilglicosídeos.

Dessa forma, neste trabalho utilizou-se a temperatura de $4^{\circ} \mathrm{C}$ durante a extração, para preservar o perfil de isoflavonas originalmente presentes na amostra. Comparou-se a eficiência de extração - 2 horas com agitador mecânico e barra magnética - de: a) $\mathrm{CH}_{3} \mathrm{CN}$ : $\mathrm{HCl} 0,1 \mathrm{~N}, 10: 2$; b) $\mathrm{CH}_{3} \mathrm{CN}: \mathrm{HCl} 0,1 \mathrm{~N}: \mathrm{H}_{2} \mathrm{O}, 10: 2: 7$; c) acetona : $\mathrm{HCl} 0,1 \mathrm{~N}, 10: 2$; d) metanol aquoso a 70 e $80 \%$; e) acetonitrila aquosa a 60 e $80 \%$. A amostra utilizada foi de farinha desengordurada de soja, já que o processo de moagem e desengorduramento sabidamente não afeta esses compostos [13]. Como as formas glicosídicas das isoflavonas apresentam quase o dobro do peso molecular das agliconas, os resultados foram normalizados para massa de agliconas, como sugerido por SONG et al [10].

De acordo com nossos resultados, as amostras extraídas com acetonitrila, tanto a 60 como a $80 \%$, quando submetidas à análise por CLAE apresentaram perfis distorcidos, não permitindo a identificação dos picos (resultados não apresentados). Tais alterações não foram observadas quando a extração com acetonitrila (ou acetona) foi realizada em meio ácido (Tabela 1), como sugerido por SONG et al [10] e WANG \& MURPHY [13].

Como podemos observar na Tabela 1, nas extrações realizadas em meio ácido, o solvente mais indicado foi acetonitrila na presença de água, que extraiu cerca de $11 \%$ a mais que a acetona e cerca de $63 \%$ a mais que a acetonitrila sem adição da água. Segundo SONG et al [10], a adição de água na proporção de 10:2:7 $\left(\mathrm{CH}_{3} \mathrm{CN}: \mathrm{HCl} 0,1 \mathrm{~N}: \mathrm{H}_{2} \mathrm{O}\right)$ melhoraria a eficiência de extração para a maioria dos produtos, mas para amostras não usuais esta proporção deveria ser otimizada [6].

TABELA 1. Extração de isoflavonas de farinha desengordurada de soja $(A)$ por diferentes

\begin{tabular}{|c|c|c|c|c|c|c|c|c|c|}
\hline & \multicolumn{3}{|c|}{$\begin{array}{c}\mathrm{CH}_{3} \mathrm{CN}: \mathrm{HCl} 0,1 \mathrm{~N}: \mathrm{H}_{2} \mathrm{O} \\
(10: 2: 7)\end{array}$} & \multicolumn{3}{|c|}{$\begin{array}{c}\mathrm{CH}_{3} \mathrm{CN}: \mathrm{HCl} 0,1 \mathrm{~N} \\
(10: 2) \\
\text { mg aglicona/100g }\end{array}$} & \multicolumn{3}{|c|}{$\begin{array}{c}\mathrm{CH}_{3} \mathrm{COCH}_{3}: \mathrm{HCl} 0,1 \mathrm{~N} \\
(10: 2)\end{array}$} \\
\hline & 1 & 2 & média & 1 & 2 & média & 1 & 2 & média \\
\hline daidzina & 21,4 & 21,4 & 21,4 & 12,1 & 12,6 & 12,4 & 20,0 & 20,4 & 20,2 \\
\hline glicitina & 1,8 & 1,8 & 1,8 & 1,0 & 0,9 & 1,0 & 1,5 & 1,5 & 1,5 \\
\hline M daidzina & 24,2 & 24,2 & 24,2 & 10,6 & 10,6 & 10,6 & 18,4 & 18,3 & 18,4 \\
\hline M glicitina & n.d. & n.d. & n.d. & n.d. & n.d. & n.d. & n.d. & n.d. & n.d. \\
\hline genistina & 49,2 & 49,8 & 49,5 & 33,7 & 34,5 & 34,1 & 45,5 & 45,4 & 45,4 \\
\hline Ac daidzina & 1,8 & 1,8 & 1,8 & 1,8 & 1,8 & 1,8 & 1,7 & 1,7 & 1,7 \\
\hline M genistina & 55,3 & 55,5 & 55,4 & 30,8 & 31,3 & 31,0 & 49,9 & 49,7 & 49,8 \\
\hline daidzeína & 6,8 & 6,9 & 6,8 & 5,7 & 5,9 & 5,8 & 6,9 & 7,0 & 7,0 \\
\hline Ac genistina & 3,9 & 4,0 & 4,0 & 3,6 & 3,8 & 3,7 & 4,0 & 4,1 & 4,0 \\
\hline gliciteína & n.d. & n.d. & n.d. & n.d. & n.d. & n.d. & n.d. & n.d. & n.d. \\
\hline genisteína & 9,0 & 9,1 & 9,1 & 7,4 & 7,7 & 7,6 & 9,0 & 9,2 & 9,1 \\
\hline TOTAL & 173,4 & 174,5 & $173,9^{\mathrm{a}}$ & 106,6 & 109,1 & $107,9^{\mathrm{b}}$ & 157,0 & 157,3 & $157,2^{\mathrm{c}}$ \\
\hline
\end{tabular}

* Foram utilizadas as abreviações $\mathrm{M}$ e Ac para os radicais malonil e acetil, respectivamente. N.d. não detectada. As extrações foram realizadas em duplicata (colunas 1 e 2). Os valores em cada coluna representam a media de duas injeções. Médias com letras diferentes são significativamente diferentes $(P<0,05)$.

Dessa forma, comparamos a eficiência da extração destes sistemas com a do metanol aquoso a $80 \%$ (Tabela 2) e observamos que este apresentou eficiência similar à do sistema $\mathrm{CH}_{3} \mathrm{CN}: \mathrm{HCl} 0,1 \mathrm{~N}: \mathrm{H}_{2} \mathrm{O}(10: 2: 7)$. As amostras extraídas com metanol, tanto $70 \%$ como $80 \%$, apresentaram bons perfis e mesmo rendimento na extração de isoflavonas; no entanto, utilizando-se metanol $80 \%$ observou-se diminuição da presença de picos contaminantes, como observado a partir da análise em CLAE (resultados não apresentados).

Comparamos também a eficiência de extração com Ultra-turrax (3 extrações de 1 minuto) e agitador mecânico (2 horas), dados que são apresentados na Tabela 2. Pode-se observar que para a farinha desengordurada de soja e o isolado protéico não houve diferença significativa entre os dois sistemas utilizados.

Analisamos também a interferência do tempo de extração, quando se utiliza o agitador mecânico, para a análise de farinha desengordurada de soja e de isolado protéico de soja. Como podemos observar na Tabela 3, extrações realizadas por 2 ou 3 horas, tanto para a farinha como para o isolado, não resultam em diferenças na quantidade total extraída. 
TABELA 2. Eficiência da extração de isoflavonas de farinha desengordurada de soja e de isolado protéico de soja em Ultra-Turrax ou agitador mecânico (2 h), com metanol aquoso a $80 \%$.

\begin{tabular}{lcccc}
\hline & \multicolumn{3}{c}{ Farinha desengordurada de soja (A) } & \multicolumn{2}{c}{ Isolado protéico de soja (A) } \\
& Ultra Turrax & \multicolumn{2}{c}{ mg de aglicona $/ 100 \mathrm{~g}$} \\
Mgitador & Ultra Turrax \\
Média $\pm \mathrm{DP}$ & Média $\pm \mathrm{DP}$ & $\begin{array}{c}\text { Agitador } \\
\text { Média } \pm \mathrm{DP}\end{array}$ & Média $\pm \mathrm{DP}$ \\
\hline daidzina & $21,9 \pm 0,6$ & $21,8 \pm 0,3$ & $5,4 \pm 0,0$ & $5,3 \pm 0,1$ \\
glicitina & $2,3 \pm 0,3$ & $2,5 \pm 0,2$ & $1,8 \pm 0,0$ & $1,8 \pm 0,1$ \\
M daidzina & $22,5 \pm 1,1$ & $21,9 \pm 0,3$ & $9,8 \pm 1,0$ & $9,9 \pm 0,9$ \\
M glicitina & $0,6 \pm 0,4$ & $0,9 \pm 0,0$ & $1,3 \pm 0,1$ & $1,3 \pm 0,1$ \\
genistina & $46,7 \pm 0,8$ & $46,2 \pm 0,6$ & $16,6 \pm 0,5$ & $16,6 \pm 0,2$ \\
Ac daidzina & $2,2 \pm 0,2$ & $2,6 \pm 0,0$ & $2,2 \pm 0,2$ & $2,0 \pm 0,2$ \\
M genistina & $58,3 \pm 1,9$ & $58,3 \pm 0,5$ & $36,1 \pm 1,0$ & $35,4 \pm 0,7$ \\
daidzeína & $6,9 \pm 0,3$ & $7,2 \pm 0,1$ & $32,7 \pm 0,4$ & $30,9 \pm 0,3$ \\
gliciteína & n.d. & n.d. & $3,2 \pm 0,1$ & $3,1 \pm 0,1$ \\
Ac genistina & $3,8 \pm 0,2$ & $4,2 \pm 0,0$ & $3,2 \pm 0,1$ & $3,1 \pm 0,1$ \\
genisteína & $9,4 \pm 0,5$ & $9,7 \pm 0,1$ & $56,3 \pm 1,2$ & $54,5 \pm 0,7$ \\
Total & $174,6 \pm 5,2^{\text {ac }}$ & $175,3 \pm 1,5^{\text {a }}$ & $168,6 \pm 4,1^{\text {bc }}$ & $163,7 \pm 2,3^{\mathrm{b}}$ \\
\hline
\end{tabular}

* Foram utilizadas as abreviações $\mathrm{M}$ e Ac para os radicais malonil e acetil, respectivamente. N. d não detectada DP = desvio-padrão; extrações realizadas em triplicata. Médias com letras iguais não apresentam diferença significativa $(P<0,05)$.

TABELA 3. Influência do tempo de extração (metanol $80 \%$ ) no teor de isoflavonas de farinha desengordurada de soja e isolado protéico de soja.

\begin{tabular}{|c|c|c|c|c|}
\hline & \multicolumn{2}{|c|}{ Farinha Desengordurada de Soja B } & \multicolumn{2}{|c|}{ Isolado Protéico de Soja B } \\
\hline & \multicolumn{4}{|c|}{$\mathrm{mg}$ de aglicona $/ 100 \mathrm{~g}$} \\
\hline & $\begin{array}{c}\text { extração por } 2 \mathbf{h} \\
\text { média } \pm \text { DP }\end{array}$ & $\begin{array}{l}\text { extração por } 3 \mathbf{h} \\
\text { média } \pm \text { DP }\end{array}$ & $\begin{array}{c}\text { extração por } \mathbf{2 h} \\
\text { média } \pm \text { DP }\end{array}$ & $\begin{array}{c}\text { extração por } 3 \mathbf{h} \\
\text { média } \pm \text { DP }\end{array}$ \\
\hline $\begin{array}{l}\text { daidzina } \\
\text { na }\end{array}$ & $27,1 \pm 1,0$ & $25,4 \pm 0,3$ & $6,3 \pm 0,0$ & $6,5 \pm 0,2$ \\
\hline glicitina & $2,2 \pm 0,3$ & $2,0 \pm 0,1$ & $2,0 \pm 0,2$ & $2,8 \pm 0,3$ \\
\hline M daidzina & $27,4 \pm 1,7$ & $24,9 \pm 0,9$ & $10,0 \pm 0,9$ & $10,0 \pm 0,8$ \\
\hline genistina & $57,9 \pm 1,8$ & $54,5 \pm 0,4$ & $19,5 \pm 0,2$ & $20,2 \pm 1,0$ \\
\hline Ac daidzina & $2,2 \pm 0,1$ & $2,0 \pm 0,0$ & $1,5 \pm 0,0$ & $1,5 \pm 0,1$ \\
\hline M genistina & $62,4 \pm 2,5$ & $61,3 \pm 0,5$ & $36,2 \pm 1,0$ & $35,9 \pm 1,2$ \\
\hline daizeína & $8,1 \pm 0,3$ & $8,0 \pm 0,1$ & $34,5 \pm 0,5$ & $34,4 \pm 1,1$ \\
\hline gliciteína & n.d. & n.d. & $3,1 \pm 0,0$ & $3,2 \pm 0,1$ \\
\hline Ac genistina & $4,8 \pm 0,2$ & $4,8 \pm 0,0$ & $4,4 \pm 0,7$ & $4,7 \pm 0,1$ \\
\hline genisteína & $11,2 \pm 0,3$ & $11,05 \pm 0,1$ & $60,4 \pm 1,0$ & $59,7 \pm 1,6$ \\
\hline TOTAL & $203,2 \pm 7,2^{\mathrm{a}}$ & $194,0 \pm 2,0^{\mathrm{a}}$ & $177,9 \pm 3,7^{\mathrm{b}}$ & $178,9 \pm 6,3^{\mathrm{b}}$ \\
\hline
\end{tabular}

* Foram utilizadas as abreviações $\mathrm{M}$ e Ac para os radicais malonil e acetil, respectivamente. DP = desvio-padrão; extrações realizadas em triplicata. N.d. não detectada. Médias com letras iguais não apresentam diferença significativa $(P<0,05)$.

Já para a análise de proteína texturizada de soja (Tabela 4), podemos observar uma acentuada diferença no teor de isoflavonas ao se utilizar o Ultra-turrax ou o agitador com barra magnética para a extração. De acordo com os resultados apresentados, conclui-se que para a proteína texturizada a melhor opção é realizar extração com agitador por 3 horas, visto que em 2 horas obteve-se um menor rendimento e desvio-padrão elevado. Essa diferença se deve, provavelmente, às condições drásticas de processamento desses produtos. Durante a extrusão, a matéria-prima (farinha desengordurada ou concentrado protéico) é submetida a pressões e temperaturas elevadas que causam alterações significativas de suas propriedades físico-quí- micas, o que poderia estar relacionado à maior dificuldade de solubilização das isoflavonas.

TABELA 4. Eficiência de extração de isoflavonas de proteína texturizada de soja.

\begin{tabular}{lccc}
\hline & Ultra-turrax & $\begin{array}{c}\text { Agitador por 2 horas } \\
\text { mg de aglicona } / 100 \mathrm{~g} \\
\text { Média } \pm \mathrm{DP}\end{array}$ & Agitador por 3 horas \\
\hline Média $\pm \mathrm{DP}$ & $3,8 \pm 0,3$ & $3,8 \pm 0,2$ & Média $\pm \mathrm{DP}$ \\
\hline glicitina & $0,9 \pm 0,0$ & $0,8 \pm 0,1$ & $5,5 \pm 0,4$ \\
M daidzina & $2,8 \pm 0,2$ & $3,7 \pm 0,3$ & $1,5 \pm 0,1$ \\
genistina & $9,5 \pm 0,7$ & $10,3 \pm 0,6$ & $5,0 \pm 0,3$ \\
Ac daidzina & $1,0 \pm 0,1$ & $1,5 \pm 0,2$ & $14,4 \pm 0,6$ \\
M genistina & $8,0 \pm 0,8$ & $10,6 \pm 0,9$ & $1,9 \pm 0,2$ \\
daidzeína & $16,1 \pm 1,7$ & $20,3 \pm 0,9$ & $14,6 \pm 0,8$ \\
gliciteína & $1,4 \pm 0,2$ & $2,5 \pm 0,2$ & $24,2 \pm 0,5$ \\
Ac genistina & $2,6 \pm 0,2$ & $2,6 \pm 0,2$ & $2,5 \pm 0,3$ \\
genisteína & $28,5 \pm 3,2$ & $35,6 \pm 1,6$ & $4,8 \pm 0,3$ \\
TOTAL & $74,6 \pm 7,1^{\mathrm{a}}$ & $91,7 \pm 5,0^{\mathrm{b}}$ & $115,9 \pm 3,1^{\mathrm{c}}$ \\
\hline
\end{tabular}

* Foram utilizadas as abreviações $\mathrm{M}$ e Ac para os radicais malonil e acetil, respectivamente. DP = desvio-padrão; extrações realizadas em triplicata. Médias com letras diferentes são significativamente diferentes $(P<0,05)$.

\section{2 - Concentração dos extratos}

$\mathrm{Na}$ determinação das melhores condições de concentração dos extratos observou-se que a temperatura de $30^{\circ} \mathrm{C}$ era inviável, já que as amostras levavam em torno de 2 horas no rotaevaporador para ter seu volume reduzido em oito vezes. Dessa forma, optamos por definir a temperatura de $40^{\circ} \mathrm{C}$ e testamos três tempos diferentes de rotaevaporação, suficientes para concentrar as amostras em 8 vezes ( 10 min), em mais de 20 vezes ( $25 \mathrm{~min}$ ), ou até secagem total (mais de $45 \mathrm{~min}$ ). As amostras concentradas foram então transferidas para balão volumétrico e o volume completado com metanol, de forma que as amostras concentradas em 8, mais de 20 vezes, ou até secagem total foram ressuspensas em metanol em concentração final de 50, 80-90 e 100\%, respectivamente. Os resultados são apresentados na Tabela 5. É importante ressaltar que amostras que permaneceram no rotaevaporador até secagem total do extrato, e que foram portanto ressuspensas em metanol puro, apresentaram perfis distorcidos quando submetidas à análise em CLAE, não sendo possível uma clara observação e identificação dos picos. Entre as amostras das farinhas concentradas em 8 e mais de 20 vezes (Tabela 5) observa-se uma diminuição no teor total de isoflavonas, e essa diferença corresponde principalmente à diminuição da malonildaidzina nas amostras concentradas em mais de 20 vezes (ressuspendidas, portanto, em metanol $80-90 \%$ ).

Estes resultados indicam que podem estar ocorrendo alterações nos compostos, como por exemplo, degradação térmica e/ou insolubilização pela complexação com outras substâncias, devido ao maior tempo de exposição à temperatura de $40^{\circ} \mathrm{C}$. Por outro lado, as amostras mais concentradas foram ressolubilizadas em uma concentração maior de metanol, o que também poderia afetar os resultados. Dessa for- 
ma, extratos de farinha desengordurada e de isolado protéico de soja foram concentrados por diferentes períodos de tempo, porém todas as amostras foram ressolubilizadas em concentração final de metanol aquoso de $50 \%$. Os resultados (Tabela 6) mostraram que não há diferenças nos teores e perfis de isoflavonas encontrados, mesmo para as amostras submetidas a secagem total. Conclui-se, portanto, que a temperatura de $40^{\circ} \mathrm{C}$ usada na concentração dos extratos não altera as amostras e que as isoflavonas são pouco solúveis em concentrações de metanol maiores que $80 \%$, provavelmente em decorrência da conjugação com compostos mais polares.

\section{3 - Filtragem das amostras}

A utilização de filtros de $0,22 \mathrm{~mm}$ ou $0,45 \mathrm{~mm}$ para a filtragem das amostras não resultou em diferenças significativas nos teores de isoflavonas encontrados através da análise em CLAE. Para a farinha desengordurada de soja $\mathrm{C}$, por exemplo, obteve-se um teor total médio de isoflavonas de $156,5 \mathrm{mg} / 100 \mathrm{~g}$, utilizando-se filtros de $0,22 \mathrm{~mm}$, e de $155,2 \mathrm{mg} / 100 \mathrm{~g}$, com filtros de $0,45 \mathrm{~mm}$. Para o isolado protéico de soja $D$ esse teor foi de $128,0 \mathrm{mg} / 100 \mathrm{~g}$, com filtro $0,22 \mathrm{~mm}$, e $129,3 \mathrm{mg} / 100 \mathrm{~g}$, com filtro de $0,45 \mathrm{~mm}$.

TABELA 5. Influência do fator de concentração dos extratos de farinha desengordurada de soja em rotaevaporador a $40^{\circ} \mathrm{C}$ (concentração final de metanol dos extratos variável).

\begin{tabular}{|c|c|c|c|c|}
\hline & \multicolumn{2}{|c|}{ Farinha C } & \multicolumn{2}{|c|}{ Farinha D } \\
\hline & \multicolumn{4}{|c|}{$\mathrm{mg}$ de aglicona $/ 100 \mathrm{~g}$} \\
\hline & \multirow{2}{*}{$\begin{array}{c}\text { concentração de 8x } \\
\text { Média } \pm \text { DP }\end{array}$} & \multirow{2}{*}{$\begin{array}{r}\text { Concentr. } \geq \mathbf{2 0 x} \\
\text { Média } \pm \text { DP }\end{array}$} & \multicolumn{2}{|c|}{ Concentração de $8 x$ Concentração $\geq 20 x$} \\
\hline & & & Média \pm DP & Média \pm DP \\
\hline 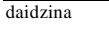 & $14,0 \pm 0,2$ & $14,9 \pm 0,2$ & $20,9 \pm 0,2$ & $22,7 \pm 0,1$ \\
\hline glicitina & $4,3 \pm 0,1$ & $4,8 \pm 0,1$ & $8,2 \pm 0,0$ & $9,4 \pm 0,0$ \\
\hline M daidzina & $32,6 \pm 0,7$ & $27,9 \pm 0,1$ & $40,7 \pm 0,2$ & $35,0 \pm 0,4$ \\
\hline M glicitina & $5,1 \pm 0,0$ & $4,4 \pm 0,0$ & $10,4 \pm 0,0$ & $8,9 \pm 0,0$ \\
\hline genistina & $21,5 \pm 0,2$ & $22,0 \pm 0,2$ & $26,3 \pm 0,2$ & $27,6 \pm 0,1$ \\
\hline Ac daidzina & $1,6 \pm 0,0$ & $1,4 \pm 0,0$ & $2,7 \pm 0,0$ & $2,6 \pm 0,0$ \\
\hline M genistina & $66,3 \pm 0,3$ & $65,0 \pm 0,3$ & $65,3 \pm 0,5$ & $63,1 \pm 0,6$ \\
\hline daidzeína & $3,3 \pm 0,1$ & $3,0 \pm 0,2$ & $3,8 \pm 0,0$ & $3,8 \pm 0,1$ \\
\hline gliciteína & n.d. & n.d. & n.d. & n.d. \\
\hline Ac genistina & $3,4 \pm 0,1$ & $3,8 \pm 0,2$ & $5,5 \pm 0,1$ & $5,3 \pm 0,1$ \\
\hline genisteína & $3,8 \pm 0,1$ & $3,7 \pm 0,2$ & $4,4 \pm 0,1$ & $4,2 \pm 0,2$ \\
\hline TOTAL & $155,9 \pm 0,7^{\mathrm{a}}$ & $151,0 \pm 0,3^{b}$ & $188,2 \pm 0,8^{\mathrm{c}}$ & $182,6 \pm 1,5^{\mathrm{d}}$ \\
\hline
\end{tabular}

Foram utilizadas as abreviações $\mathrm{M}$ e Ac para os radicais malonil e acetil, respectivamente. DP = desvio-padrão.

As amostras concentradas 8 vezes foram ressolubilizadas em metanol aq. de forma a obter concentração final de 50\%; as amostras concentradas em mais de 20 vezes foram ressolubilizadas em metanol aq. de forma a obter concentração final entre $80-90 \%$. N.d. não detectada. Médias com letras diferentes são significativamente diferentes $(P<0,05)$.

Foram utilizadas as abreviações $M$ e Ac para os radicais malonil e acetil, respectivamente. As extrações foram realizadas em duplicata, sendo um dos extratos submetido à secagem total e o outro até a concentração indicada. Os valores representam a média de duas injeções. Médias com letras diferentes são significativamente diferentes $(P<0,05)$.

TABELA 6. Influência do fator de concentração dos extratos em rotaevaporador a $40^{\circ} \mathrm{C}$ (concentração final de metanol dos extratos ajustada para $50 \%$ ).

\begin{tabular}{lcccc}
\hline & \multicolumn{3}{c}{ Farinha D } & \multicolumn{3}{c}{ Isolado C } \\
& conc. 20x & até secagem & conc. 8x & até secagem \\
\hline daidzina & 26,0 & 24,8 & 5,6 & 5,6 \\
glicitina & 10,9 & 10,8 & 3,9 & 4,0 \\
M daidzina & 39,7 & 43,1 & 18,1 & 17,9 \\
M glicitina & 10,8 & 10,7 & 2,7 & 2,8 \\
genistina & 29,0 & 28,6 & 13,4 & 13,5 \\
Ac daidzina & 3,0 & 2,9 & 2,1 & 2,1 \\
M genistina & 59,1 & 58,1 & 44,5 & 44,2 \\
daidzeína & 3,8 & 3,8 & 15,8 & 15,7 \\
gliciteína & 1,0 & 1,0 & 2,6 & 2,6 \\
Ac genistina & 5,0 & 4,8 & 5,7 & 5,7 \\
genisteína & 4,2 & 4,0 & 24,1 & 23,1 \\
TOTAL & $192,4^{\mathrm{a}}$ & $192,5^{\mathrm{a}}$ & $138,4^{\mathrm{b}}$ & $137,2^{\mathrm{b}}$ \\
\hline
\end{tabular}

\section{4 - Teste de recuperação}

O método de extração e preparo de amostra proposto, com metanol aquoso a $80 \%\left(2 \mathrm{~h} / 4^{\circ} \mathrm{C}\right)$, teve a sua recuperação testada para amostras de farinha desengordurada e isolado protéico de soja adicionadas dos padrões de daidzina e genistina. A partir da Tabela 7 observa-se que a recuperação de daidzina variou entre 98,5 a $103,3 \%$ e a de genistina, entre 95,4 a $102,7 \%$, demonstrando portanto a sua adequabilidade.

TABELA 7. Recuperação de daidzina e genistina adicionadas a amostras de farinha desengordurada e isolado protéico de soja.

\begin{tabular}{lcc}
\hline & daidzina & genistina \\
\hline Farinha & & \\
Teor presente $(\mathrm{mg} / 100 \mathrm{~g})$ & $24,4 \pm 1,8$ & $92,6 \pm 3,1$ \\
Teor adicionado $(\mathrm{mg} / 100 \mathrm{~g})$ & 39,7 \\
Teor encontrado $(\mathrm{mg} / 100 \mathrm{~g})$ & $64,7 \pm 0,8$ & $126,1 \pm 1,0$ \\
Recuperação $(\%)$ & $98,5 \pm 1,3$ & $95,4 \pm 0,7$ \\
Isolado & & $31,2 \pm 0,3$ \\
Teor presente $(\mathrm{mg} / 100 \mathrm{~g})$ & $10,3 \pm 0,1$ & 39,7 \\
Teor adicionado $(\mathrm{mg} / 100 \mathrm{~g})$ & 21,4 & $72,8 \pm 1,1$ \\
Teor encontrado $(\mathrm{mg} / 100 \mathrm{~g})$ & $32,7 \pm 0,7$ & $102,7 \pm 1,5$ \\
\% Recuperação & $103,3 \pm 2,2$ & \\
\hline
\end{tabular}

\section{5 - Hidrólise para obtenção das agliconas}

Alguns métodos para quantificação de isoflavonas se baseiam na obtenção prévia destes compostos na 
forma de agliconas, através de uma hidrólise ácida. Para efeito de comparação, as diferentes condições de hidrólise foram testadas, para a farinha desengordurada de soja. O método de WANG et al [12], baseado na hidrólise da amostra em $\mathrm{HCl} 1 \mathrm{~N}$ aquoso (refluxo 2h) com posterior extração das agliconas em acetonitrila, resultou na presença de vários picos contaminantes e de genistina, indicando que a hidrólise não havia sido suficiente. Já o método de FRANKE et al [4], que realiza extração da amostra e hidrólise concomitantes, com $\mathrm{HCl} 2 \mathrm{~N}$ em etanol (refluxo $2 \mathrm{~h}$ ), resultou em poucos contaminantes, porém os picos apresentaram-se assimétricos (Figura $1 D$ ).

Os melhores resultados foram obtidos ao realizar a hidrólise das isoflavonas após sua extração em metanol ou etanol $80 \%$, como sugerido por PETERSSON \& KIESSLING [7], quando se observou a presença de apenas um pico contaminante, porém que não se sobrepôs aos picos referentes às agliconas (Figura 1B). Esse pico não foi retido em coluna de poliamida demonstrando não tratar-se de isoflavonas (Figura 1C). No entanto, o teor de agliconas totais obtido através da hidrólise do extrato (Tabela 8) é menor do que o obtido pela conversão dos resultados das amostras não hidrolisadas em agliconas. Além disto, a hidrólise por 1 hora foi insuficiente, já que ainda se detectou a presença de genistina. O aumento do tempo de hidrólise para 2 horas foi eficiente, porém obteve-se um teor total de agliconas ainda menor. Esses resultados indicam que as isoflavonas sofreram degradação durante o processo de hidrólise. Nota-se ainda que a proporção entre daidzeína e genisteína após a hidrólise foi de 1:1,56, enquanto que na amostra não hidrolisada foi de 1:2,32, o que indica que o composto que se degrada com maior facilidade é a genisteína.

TABELA 8. Influência do tempo de hidrólise $(\mathrm{HCl} 2 \mathrm{~N})$ para a obtenção de agliconas, a partir do extrato etanólico da farinha desengordurada de soja.

\begin{tabular}{lcc}
\hline & agliconas totais (mg/100g) & \% perda \\
\hline Sem hidrólise & 208,9 & 0 \\
Hidrólise por 1 h & 153,7 & 26,4 \\
Hidrólise por 2 h & 133,8 & 36,0 \\
\hline
\end{tabular}

A partir destes resultados conclui-se que os métodos baseados na quantificação de agliconas subestimam o teor de isoflavonas, devido à degradação ocorrida durante a hidrólise ácida. Desta forma, a determinação de isoflavonas na forma dos glicosil-derivados originalmente presentes mostra-se mais adequada, além de dar informações sobre o processamento ao qual os alimentos foram submetidos.

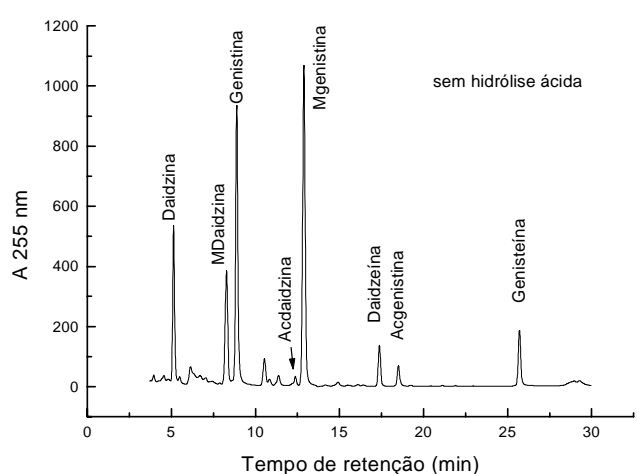

A

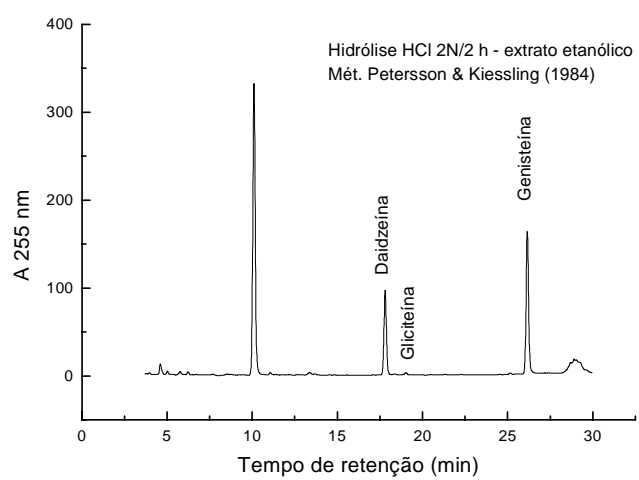

B

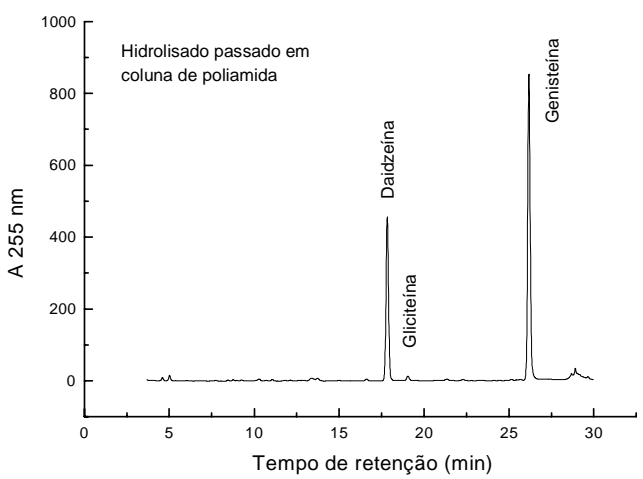

C

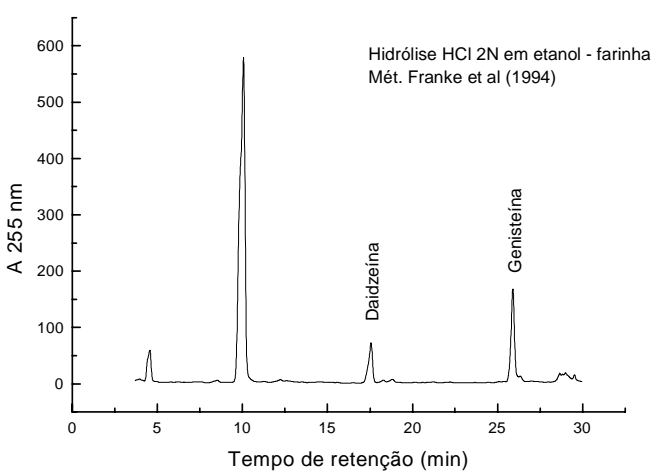

FIGURA 1. Cromatogramas obtidos por CLAE de extratos de farinha desengordurada de soja submetidos ou não à hidrólise ácida. Solvente $\mathrm{A}$ : ácido acético $0,1 \%$ em água; solvente $\mathrm{B}$ : ácido acético $0,1 \%$ em acetonitrila. A fase móvel consistiu em gradiente linear de acetonitrila em fluxo de $\mathrm{mL} / \mathrm{min}$. A) Extrato etanólico; B) Extrato etanólico hidrolisado $(\mathrm{HCl} 2 \mathrm{~N} / 2 \mathrm{~h})$ pelo método de PETERSSON \& KIESSLING [7]; C) Hidrolisado obtido em B passado em coluna de poliamida; D) Farinha extraída em $\mathrm{HCl}$ 2N etanólico pelo método de Franke et al [4]. 


\section{6 - Extração em fase sólida}

Avaliaram-se as condições ideais para extração em fase sólida, visando a determinação de isoflavonas em produtos alimentícios nos quais os teores muito baixos e/ou a presença de interferentes impossibilitariam a análise direta dos extratos metanólicos concentrados. Os resultados mostraram a ausência de malonilglicosídeos nos eluatos metanólicos das colunas de poliamida que, conforme determinado posteriormente, também são retidos porém necessitam de $\mathrm{pH}$ alcalino para sua eluição (Tabela 9). Mesmo assim, observou-se diminuição de aproximadamente 14\% do teor total de isoflavonas quando comparado com os extratos que não foram passados em coluna. Testes similares realizados com colunas $\mathrm{C} 18$ mostraram recuperação de cerca de $82 \%$ das isoflavonas, sendo que o restante foi encontrado na água de lavagem. Estes resultados indicaram a saturação da capacidade das colunas, o que foi confirmado ao se utilizar a metade do volume dos extratos, obtendo-se recuperação de $100 \%$.

Desta forma, conclui-se que a capacidade de ligação das colunas de poliamida e C18 gira em torno de 1,4 a 1,8mg em agliconas por grama de fase sólida. No caso das farinhas desengorduradas de soja isso equivaleria a utilizar $0,5 \mathrm{~g}$ de amostra para cada $\mathrm{g}$ de poliamida ou C18, para garantir que haja a ligação total das isoflavonas.

TABELA 9. Eficiência do metanol como solvente de eluição na extração em fase sólida (coluna de poliamida) das isoflavonas de farinhas desengorduradas de soja.

\begin{tabular}{lcccc}
\hline mg aglic/100g & $\begin{array}{c}\text { Controle } \\
(\mathbf{A})\end{array}$ & $\begin{array}{c}\text { Eluato } \mathbf{M e O H} \\
(\mathbf{A})\end{array}$ & $\begin{array}{c}\text { Controle } \\
(\mathbf{B})\end{array}$ & $\begin{array}{c}\text { Eluato (B) } \\
\mathbf{M e O H}: \mathbf{N H}_{3}\end{array}$ \\
\hline daidzina & 22,2 & 19,4 & 29,1 & 27,9 \\
glicitina & 2,6 & 1,6 & 2,7 & 2,2 \\
M daidzina & 25,1 & n.d. & 22,1 & 17,7 \\
M glicitina & n.d. & n.d. & n.d. & n.d. \\
genistina & 53,2 & 43,8 & 71,0 & 66,3 \\
Ac daidzina & 2,7 & 2,1 & 2,6 & 1,5 \\
M genistina & 58,5 & n.d. & 57,1 & 44,2 \\
daidzeína & 7,8 & 7,2 & 8,3 & 7,6 \\
Ac genistina & 5,2 & 4,4 & 4,9 & 3,2 \\
genisteína & 11,0 & 9,6 & 11,2 & 10,0 \\
TOTAL & 188,3 & 88,1 & 208,9 & 180,6 \\
\hline
\end{tabular}

* Foram utilizadas as abreviações $\mathrm{M}$ e Ac para os radicais malonil e acetil, respectivamente. Os controles consistiram dos extratos não submetidos à extração em fase sólida. n.d. $=$ não detectado. Proporção metanol:amônia $=99,5: 0,5$

\section{4 - CONCLUSÕES}

- O melhor solvente para extração, em termos de eficiência e praticidade, é o metanol $80 \%$.

- Não há diferenças significativas entre a eficiência das extrações realizadas com Ultra-Turrax ou com agitador mecânico, durante 2 ou 3 horas, para farinhas e isolados, enquanto que para produtos processados em condições mais severas (texturizados) a extração mais eficiente é conseguida aumentando-se o tempo de extração em agitador para 3 horas.

- A temperatura de $40^{\circ} \mathrm{C}$ durante a concentração dos extratos, por rotaevaporação, não altera a amostra. Para injeção no cromatógrafo líquido, o volume do extrato aquoso deve ser ajustado com metanol a fim de obter-se uma concentração final entre 50 e $80 \%$.

- Não há diferenças significativas nos teores de isoflavonas decorrentes da utilização de filtros de amostra de $0,22 \mathrm{~mm}$ ou $0,45 \mathrm{~mm}$.

- Para que haja hidrólise total das isoflavonas é necessário um refluxo de 2 horas com $\mathrm{HCl} 2 \mathrm{~N}$ em banho de água fervente, porém as isoflavonas sofrem degradação nestas condições.

- O melhor solvente para a eluição de isoflavonas e seus glicosil-derivados, após passagem da amostra por coluna de extração em fase sólida, é $\mathrm{MeOH}: \mathrm{NH}_{3}(99,5: 0,5)$.

- As colunas de Poliamida e de C18 apresentam capacidade de ligação de isoflavonas em torno de 1,4 a 1,8mg expressos em agliconas por grama de fase sólida.

\section{5 - REFERÊNCIAS BIBLIOGRÁFICAS}

[1] BARNES, S.; KIRK, M.; COWARD, L. Isoflavones and their conjugates in Soy Foods: Extraction conditions and Analysis by HPLC - Mass Spectometry. J. Agric. Food Chem., v. 42, p. 2466-2474, 1994.

[2] COWARD, L.; SMITH, M.; KIRK, M.; BARNES, S. Chemical modification of isoflavones in soyfoods during cooking and processing. Am. J. Clin. Nutr., v. 68, p. 1486S-1491S, 1998.

[3] FARMAKALIDIS, E.; MURPHY, P.A. Isolation of 6"'-OAcetylgenistin and 6"-O-Acetyldaidzin from toasted defatted soyflakes. J. Agric. Food Chem., v. 33, p. 385-389, 1985.

[4] FRANKE, A.A; CUSTER, L.J.; CERNA, C.M.; NARALA, K.K. Quantitation of phytoestrogens in legumes by HPLC. J. Agric. Food Chem., v. 42, p. 1905-1913, 1994.

[5] KUDOU, S.; FLEURY, Y.; WELTI, D.; MAGNOLATO, D.; UCHIDA, T.; KITAMURA, K.; OKUBO, K. Malonyl isoflavone glycosides in soybean seeds (Glycine max Merrill). Agric. Biol. Chem., v. 55, p. 2227-2233, 1991.

[6] MURPHY, P.A.; SONG, T.; BUSEMAN, G.; BARUA, K.; BEECHER, G.R.; TRAINER, D.; HOLDEN, J. Isoflavones in retail and institucional soy foods. J. Agric. Food Chem., v. 47, p. 2697-2704, 1999.

[7] PETTERSON, H.; KIESSLING, K.-H. Liquid Chromatographic Determination of the Plant Estrogens Coumestrol and Isoflavones in Animal Feed. J. Assoc. Off. Anal. Chem., v. 67, p. 503-506, 1984.

[8] RHODES, M.J.C. Physiologically-active compounds in plant foods: an overview. Proc. Nutr. Soc., v. 55, p. 371-384, 1996.

[9] SETCHELL, K.D.R.; CASSIDY, A. Dietary isoflavones: biological effects and relevance to human health. J. Nutr., v. 129, 758S-767S, 1999.

[10] SONG, T.; BARUA, K.; BUSEMAN, G.; MURPHY, P.A. Soy isoflavones analysis: quality control and a new internal standard. Am. J. Clin. Nutr., v. 68, p. 1474S-1479S, 1998. 
[11] THAM, D.M.; GARDENER, C.D.; HASKELL, W.L. Potential health benefits of dietary phytoestrogens: a review of the clinical, epidemiological, and mechanistic evidence. J. Clin. Endocr. Metab., v. 83, p. 2223-2235, 1998.

[12] WANG, G.; KUAN, S.S.; FRANCIS, O.J.; WARE, G.M.; CARMAN, A.S. A Simplified HPLC Method for the Determination of Phytoestrogens in Soybean and Its Processed Products. J. Agric. Food Chem., v. 38, p. 185-190, 1990.
[13] WANG, H.; MURPHY, P.A. Isoflavone Content in Commecial Soybean Foods. J. Agric. Food Chem., v. 42, p. 16661673, 1994.

\section{6 - AGRADECIMENTOS}

À FAPESP, pelo financiamento do projeto de pesquisa, e à estagiária de Iniciação Científica Fabiana Ribeiro Salerno (Bolsista CNPq) pelo auxílio na execução das análises. 